\title{
Moieties in antidiabetic drugs as a target of insulin receptors in association with common neurological disorders (Review)
}

\author{
DAVID CALDERÓN GUZMÁN ${ }^{1 *}$, HUGO JUÁREZ OLGUÍN², \\ ERNESTINA HERNÁNDEZ GARCÍA ${ }^{2 *}$, MARIBEL ORTIZ HERRERA ${ }^{3 *}$ \\ and NORMA OSNAYA BRIZUELA ${ }^{1 *}$
}

\begin{abstract}
Laboratories of ${ }^{1}$ Neurochemistry, ${ }^{2}$ Pharmacology and ${ }^{3}$ Experimental Bacteriology, National Institute of Pediatrics, Faculty of Medicine, National Autonomous University of Mexico, Mexico City, CP 04530, Mexico
\end{abstract}

Received September 22, 2015; Accepted February 18, 2016

DOI: $10.3892 /$ br.2016.616

\begin{abstract}
Insulin is a peptide that can be harmful with regards to neuroplasticity, neuroprotection and neuromodulation. Furthermore, the role of insulin highlights its relevance in the progress of diverse clinical disorders as well as in the mechanisms associated with certain pathogenesis and their evolution towards diabetes, obesity and neurodegenerative diseases. The precise molecular mechanisms by which these diseases are induced remain to be elucidated. The benefits in knowing/discovering these mechanisms in animal models and humans cannot be undermined. An in depth understanding of the principal risk factors leading to obesity and their management is vital in the implementation of early-life strategies of intervention and prevention, with a view to avoid adverse late-life outcomes. Therefore, the aim of the present study was to review their possible association with antidiabetic drugs.
\end{abstract}

\section{Contents}

1. Introduction

2. Insulin and obesity

3. Pathogenesis of oxidative stress in the brain

4. Obesity and Alzheimer's disease

5. Diabetes and drug treatments

6. Expectance

Correspondence to: Dr Hugo Juárez Olguín, Laboratory of Pharmacology, National Institute of Pediatrics, Faculty of Medicine, National Autonomous University of Mexico, Av Imán no. 1, 3er piso, Col Cuicuilco, Mexico City, CP 04530, Mexico

E-mail: juarezol@yahoo.com

${ }^{*}$ Contributed equally

Key words: obesity, insulin, antidiabetic drugs, neurodegenerative disorders, central nervous system

\section{Introduction}

Certain regions of the brain, particularly the brainstem as well as the hypothalamus, are known to be the homeostatic control centers of feeding, glucose homeostasis and energy consumption. Such brain spaces are composed of neurons and neuronal circuits, which may directly or indirectly be activated or inhibited by lipids, glucose or amino acids. An imbalance in the ability of the neurons to detect the signals emitted by such nutrients could provoke metabolic diseases such as obesity and diabetes (1). The brain is responsible for the two poles of glycemia (hyperglycemia and hypoglycemia) and the cells that detect glucose are located in numerous anatomical regions, such as the central nervous system (CNS). The regulation of glucose homeostasis and the sum of energy reaching the neurocircuits of the hypothalamus and those of higher brain regions, such as the dopaminergic system, is the function of the insulin-sensing cells of the CNS. Hyperinsulinemia of a fetus is principally in response to bad maternal nutritional habit that could lead to obesity and/or diabetes. This could cause a disturbance in the neurocircuit development in the fetus thereby disposing the fetus to metabolic diseases later in life (2).

\section{Insulin and obesity}

Obesity has been described as a New World Syndrome (3). It is characterised by an excess of glucose and body fat, which, the majority of the time, results in an increase in body weight. Obesity is defined as a body mass index $\geq 30 \mathrm{~kg} / \mathrm{m}^{2}$ in adults (4). Changes in glucose levels mobilize the neuroendocrine response in charge of preventing and correcting glycemia. The hypothalamus is the main area of the brain responsible for regulating glycemic homeostasis. Consequently, metabolic diseases such as diabetes and obesity arise principally as a result of imbalance in this control (5). Zeng et al (6), in a study of fatty acid metabolism using Yhhu981, a powerful novel compound that functions to stimulate oxidation of fatty acid and to exert pleiotropic effects on lipid metabolism (by activating $5^{\prime}$ adenosine monophosphate-activated protein kinase), suggested that defects in the metabolism of fatty acid contributed to the pathogenesis of insulin resistance and obesity. Similarly, maternal obesity present during pregnancy 
could modify the development of certain specific fetal brain cell-networks. These defects can produce pathologies, such as metabolic syndrome and possibly certain neurological diseases, in the offspring at a later age (7).

In epidemiological studies, the level of adiposity is linked to dementia and Alzheimer's disease (AD). Overweight and obesity in mid and late-life may increase the risk of dementia (8). Currently, these disorders are considered as potential risk factors that could lead to several neurodegenerative failures (9).

Environmental factors existing during pregnancy and the postnatal period could have an impact on stress factors, susceptibility genes and brain development in a structural and functional manner, giving rise to disorders that could manifest later in life. Aging produces a desynchronization of biological systems which critically worsens brain entropy/decline. In AD, this imbalance may affect cortisol, noradrenaline, stress components and reactive oxygen species (ROS), the result of which is membrane damage and an insulin-resistant brain state leading to a decrease in glucose/energy metabolism (10). Presently, it has been discovered that treatments with cancer therapeutic agents such as glucocorticoids, chemotherapy, hormonal therapies and targeted drugs can induce insulin resistance (11).

The neurological systems responsible for the identification and response to salient stimuli are important for the survival in difficult and unsteady environmental conditions. Differences among humans, such as variations in genetics, and hormonal as well as metabolic status involve behavioral strategies and neuronal responses to changes in the environment. Certain investigators suggest that the capacity of leptin in promoting stress-induced dopaminergic function is crucial in the production of pathological states including mood, disorders in the use of drugs and eating promoting obesity, where dopamine has an important role (12). The study by Ariaans et al (11) indicates that diabetes is associated with reduced basal dopamine levels in the nucleus accumbens. Possibly, the free radicals in the CNS are responsible for the induction of these disorders (13).

\section{Pathogenesis of oxidative stress in the brain}

The amount of free radicals generated should be in equilibrium with the antioxidant system. Disruption of this equilibrium would give rise to cell damage, elicit disorders in the physiological state and foster pathological process such as neurodegenerative disorders, and DNA 8-oxo-7,8-dihydroguanine accumulation due to the integration of oxidized nucleotide resulting from replication or due to the oxidation of DNA guanine (14). Similarly, the mitochondrial respiratory chain becomes the principal site of superoxide radical production. However, the precise mechanism as well as the exact location for the generation of ROS within the mitochondrial respiratory chain, is yet to be elucidated. Oxidative stress has been shown to constitute a key factor for the onset of neurodegenerative diseases (15). Excessive production of ROS or reactive nitrogen species (RNS) is proposed as detrimental to target cells, and may be responsible for diverse degenerative processes of certain human diseases in the CNS. Depending on the species or target cells, ROS or RNS may be beneficial or harmful in neuronal signaling pathways concerned with the pathophysiology of the neurodegenerative diseases (16).
By contrast, neurodegeneration is characterized by selective neuron loss. In the brain regions, the loss of neurons in the hippocampus and substantia nigra sites are known to be extremely vulnerable to cell damage, and this is attributed to $\mathrm{AD}$ (17). In sporadic AD, disorders of metabolism such as atherosclerosis, diabetes or obesity [products of hypercholestrolaemia (Hpc)] constitute an important risk factor. Hpc is associated with an increment in immunoglobulin $\mathrm{G}$ that acts against oxidized lipoproteins. The Fc region of the immunoglobulin $\mathrm{G}$ has specific receptors and it has been found that in $\mathrm{AD}$, autoantibodies against these receptors and non-brain antigens are produced. The $\gamma$-chain is the principal subunit that activates $\mathrm{Fc}$ receptors and it has been found that in rats, deletion of this chain has a protective effect against learning and memory disorders and does not increase cholesterolaemia nor affect the level of brain serum immunoglobulin G. Such protective effects are due to a decrease in tau hyperphosphorylation, loss of synapsis and accumulation of intracellular amyloid $\beta(A \beta)$ not only in the neurons of the cortex but also in the hippocampal pyramid. The receptors of the Fc region of immunoglobulin $\mathrm{G}$ has an important role in the development of AD-hpc-associated features suggesting a new potential target in the prevention of $\mathrm{AD}$ in patients with $\mathrm{Hpc}$ (18).

Adult neural stem cells (NSCs) exist in few regions of the brain. In adult rats, the multipotent version of this cell, the htNSCs, are found in the mediobasal hypothalamus. Consumption of high fat-containing food in the chronic form is capable of reducing these cells, as well as activating IкB kinase $\beta(\mathrm{IKK} \beta) /$ nuclear factor- $\mathrm{\kappa B}(\mathrm{NF}-\kappa \mathrm{B})$ linked to htNSCs. It has been proposed that adult htNSCs are crucial in the regulation of metabolic physiology and that IKK $\beta / \mathrm{NF}-\mathrm{kB}$-mediated impairment of adult htNSCs is an important neurodegenerative mechanism for the development of obesity and associated diabetes (19).

Epidemiological data suggests an increased risk of developmental dementia in individuals with obesity and type 2 diabetes and also in those with poor insulin sensitivity without diabetes, which causes a mechanistic link between adiposity, insulin sensitivity and dementia. Diabetes can cause neurodegenerative diseases due to its effect on the actions of insulin in the neurons (20).

\section{Obesity and Alzheimer's disease}

Insulin resistance by the central nervous regions could lead to obesity and AD. Such resistance may be as a result of genetic polymorphisms or due to long-term exposure to high amounts of circulating insulin as a result of insulin resistance at the peripheral level (21). Such disorder is possibly produced by toxic lipids and ceramides that cross the blood-brain barrier through a liver-brain axis. Once in the brain spaces, the resultant effects are brain insulin resistance, oxidative stress and neuro-inflammation (22). Any injury to the body produces an inflammation principally due to the innate immune response and through the systemic circulation, the inflammatory reaction is propagated to the CNS. In chronic conditions and in obesity as well as diabetes, this type of event can be observed. Inflammation in the CNS contributes to the pathogenesis of neurodegenerative disorders principally $\mathrm{AD}$, multiple sclerosis and Parkinson's disease. These disorders are prone to exacerbation as a result of increased inflammation 
Table I. Novel and previous antidiabetic drugs used to improve blood glucose levels.

\begin{tabular}{ll}
\hline Drug & \multicolumn{1}{c}{ Effects } \\
\hline Metformin & $\begin{array}{l}\text { Principal therapy for T2D. Increases adenosine monophosphate-activated protein } \\
\text { kinase activity } \\
\text { SGLT2 inhibitor with a mechanism of action that is independent of } \beta \text {-cell function and } \\
\text { the insulin pathway } \\
\text { Improves glycemic control with a lower risk of hypoglycemia and weight gain in } \\
\text { Sitagliptin }\end{array}$ \\
$\begin{array}{l}\text { diabetic patients } \\
\text { Imeglimin }\end{array}$ & $\begin{array}{l}\text { Potent and competitive SGLT2 inhibitor for the management of T2D } \\
\text { Luseogliflozin }\end{array}$ \\
Canagliflozin and dapagliflozin & $\begin{array}{l}\text { New SGLT2 inhibitors for management of T2D } \\
\text { Ipragliflozin }\end{array}$
\end{tabular}

T2D, type 2 diabetes; SGLT2, sodium glucose co-transporter-2.

within the CNS, following peripheral injury (23). Although obesity is linked to structural brain changes, little is known regarding these associations with the rate of brain atrophy. The only major decline was observed in the volume of gray matter, precuneus, cingulate and orbito-frontal gyro in globally obese people. Midlife obesity may be an important modifier of brain atrophy in individuals who are developing cognitive impairment and dementia, but in demented older adults, it has little effect on structural brain integrity (24). Metabolic disturbances such as insulin resistance, diabetes, obesity and neuropsychiatric diseases have been demonstrated in human and animal studies, which suggest the likelihood of sharing the same pathophysiological mechanisms. As a pleiotropic peptide, insulin is associated with neuroplasticity, neurotrophism and neuromodulation. Furthermore, this compound has an important role in the progression of various neuropsychiatric diseases, such as the mechanisms associated with the pathogenesis and evolution of obesity, diabetes and neurodegenerative problems such as AD (25).

The neurotrophic effect of insulin is clearly manifested at moderate concentrations. Reduction in the clearance of $A \beta$ in brain regions is possibly due to the action of insulin, for the fact that the two share a common and depurative mechanism: The insulin-degrading enzyme (IDE). IDE is more selective for insulin than for $\mathrm{A} \beta$, hence, brain hyperinsulinism may exclude $A \beta$ proof from its principal clearance mechanism (26).

Insulin is the most effective pharmacological treatment for the control of hyperglycemia, and the main target tissue is the liver (27). Insulin resistance leads to hyperinsulinemia and eventually hyperglycemia. In the United States, the diabetic rate has continued to rise. It is estimated that $>25$ million people in the United States currently have either T1D or T2D (28).

\section{Diabetes and drug treatments}

Despite advances in detection and insulin therapies, the prevalence of diabetes has continued to increase. The choice of an insulin treatment, timely initiation and schedule of insulin therapy are crucial factors in reaching optimal glycemic control. This involves a proper combination of insulin and antidiabetic agents in a way that guarantees safety and improves blood glucose levels (Table I) (29-35).

The chemical structure of metformin is open with numerous electrophilic atoms, while that of Imeglimin is a cyclic structure. The two drugs have similar moieties and consist of five nitrogen atoms. The other structures, including potent antidiabetic agents, are large and contain phenyls and pyrans moieties C-6 substituted with methyl, fluorine and chlorine atoms that suggest abundant steric effects in the receptor tissues.

Regardless of the intensive glycemic control, mortality in diabetic patients with cardiovascular diseases has not reduced. This poses a significant challenge indicating that a scheme of strict glycemic control in the management of diabetes is required. The use of sulfonylurea is significantly associated with severe hypoglycemia in patients with type 2 diabetes treated with insulin. However, the use of biguanide (45-76\%) and thiazolidinediones (15-34\%) is associated with the development of severe hypoglycemia (36).

Currently, there are $\sim 9$ different oral pharmaceutical classes and several insulin and noninsulin injectable medications for the treatment of T2D (37). The antidiabetic drugs have widely been used as first-line antidiabetic medicines for the treatment of T2D and for suppressing hepatic glucose production. The latter is the main mechanism by which metformin improves hyperglycemia through the suppression of gluconeogenesis and stimulation of glycolysis (38).

Insulin prevents synaptic deterioration which is the basis of severe memory loss in early AD (39). Insulin signaling impairment in the brain could promote the formation of neurofibrillary tangles with abnormally hyperphosphorylated tau protein (40), and a reduction in tyrosine hydroxylase in the brain has been found to occur in this disease (41). Similarly, the effects of metformin were found to be antagonized by the addition of insulin, which reduced $A \beta$ levels, oxidative stress, mitochondrial dysfunction and eventually cell death (42).

\section{Expectance}

It appears that adiposity is instrumental in Parkinson's disease and AD. The development of obesity is a result of early-life events. There may an association between early determinants 
of obesity, as insulin resistance is associated with the development of these neurological disorders (43). Treatments aimed at preventing $\beta$-cell loss or increasing the number of $\beta$-cells may inhibit the progression of diabetes and enhance the restoration of normal metabolism, and the targets for this are $\beta$-cell proliferation, neogenesis and survival (44). An integral knowledge of the key risk factors involved in obesity and their management is vital in the implementation of early-life strategies, intervention and prevention, with a view to avoid adverse outcomes later in life.

\section{Acknowledgements}

The authors would like to thank Dr Cyril Ndidi Nwoye, a native English speaker and language professor, for the critical review and translation of this manuscript. The manuscript was checked and certificated for Language \& Language editing by a Manuscript Proofreading Services.

\section{References}

1. Thorens B: Sensing of glucose in the brain. Handbook Exp Pharmacol 209: 277-294, 2012.

2. Vogt MC and Brüning JC: CNS insulin signaling in the control of energy homeostasis and glucose metabolism - from embryo to old age. Trends Endocrinol Metab 24: 76-84, 2013.

3. Nammi S, Koka S, Chinnala KM and Boini KM: Obesity: An overview on its current perspectives and treatment options. Nutr J 3: 3, 2004.

4. Taylor RW, Keil D, Gold EJ, Williams SM and Goulding A: Body mass index, waist girth, and waist-to-hip ratio as indexes of total and regional adiposity in women: Evaluation using receiver operating characteristic curves. Am J Clin Nutr 67: 44-49, 1998.

5. Camargo RL, Torrezan R, de Oliveira JC, Branco RC, Barella LF, Grassiolli S, Gravena C and Mathias PC: An increase in glucose concentration in the lateral ventricles of the brain induces changes in autonomic nervous system activity. Neurol Res 35: $15-21,2013$.

6. Zeng HL, Huang SL, Xie FC, Zeng LM, Hu YH and Leng Y: Yhhu981, a novel compound, stimulates fatty acid oxidation via the activation of AMPK and ameliorates lipid metabolism disorder in ob/ob mice. Acta Pharmacol Sin 36: 343-352, 2015.

7. Stachowiak EK, Oommen S, Vasu VT, Srinivasan M, Stachowiak M, Gohil K and Patel MS: Maternal obesity affects gene expression and cellular development in fetal brains. Nutr Neurosci 16: 96-103, 2013.

8. Gustafson DR: Adiposity and cognitive decline: Underlying mechanisms. J Alzheimers Dis 30 (Suppl 2): S97-S112, 2012

9. Sriram K, Benkovic SA, Miller DB and O'Callaghan JP: Obesity exacerbates chemically induced neurodegeneration. Neuroscience 115: 1335-1346, 2002.

10. Salkovic-Petrisic M, Osmanovic J, Grünblatt E, Riederer P and Hoyer S: Modeling sporadic Alzheimer's disease: The insulin resistant brain state generates multiple long-term morphobiological abnormalities inclusive hyperphosphorylated tau protein and amyloid-beta. J Alzheimers Dis 18: 729-750, 2009.

11. Ariaans G, de Jong S, Gietema JA, Lefrandt JD, de Vries EG and Jalving M: Cancer-drug induced insulin resistance: Innocent bystander or unusual suspect. Cancer Treat Rev 41: 376-384, 2015.

12. Burghardt PR, Love TM, Stohler CS, Hodgkinson C, Shen PH, Enoch MA, Goldman D and Zubieta JK: Leptin regulates dopamine responses to sustained stress in humans. J Neurosci 32: 15369-15376, 2012.

13. Coyle JT and Puttfarcken P: Oxidative stress, glutamate, and neurodegenerative disorders. Science 262: 689-695, 1993.

14. De Luca G, Russo MT, Degan P, Tiveron C, Zijno A, Meccia E, Ventura I, Mattei E, Nakabeppu Y, Crescenzi M, et al: A role for oxidized DNA precursors in Huntington's disease-like striatal neurodegeneration. PLoS Genet 4: e1000266, 2008.

15. Adam-Vizi V: Production of reactive oxygen species in brain mitochondria: Contribution by electron transport chain and non-electron transport chain sources. Antioxid Redox Signal 7: 1140-1149, 2005.
16. Calabrese V, Sultana R, Scapagnini G, Guagliano E, Sapienza M, Bella R, Kanski J, Pennisi G, Mancuso C, Stella AMG, et al: Nitrosative stress, cellular stress response, and thiol homeostasis in patients with Alzheimer's disease. Antioxid Redox Signal 8: 1975-1986, 2006.

17. Okouchi M, Ekshyyan O, Maracine M and Aw TY: Neuronal apoptosis in neurodegeneration. Antioxid Redox Signal 9: 1059-1096, 2007.

18. Fernandez-Vizarra P, Lopez-Franco O, Mallavia B, Higuera-Matas A, Lopez-Parra V, Ortiz-Muñoz G, Ambrosio E, Egido J, Almeida OF and Gomez-Guerrero C: Immunoglobulin $G$ Fc receptor deficiency prevents Alzheimer-like pathology and cognitive impairment in mice. Brain 135: 2826-2837, 2012.

19. Li J, Tang Y and Cai D: IKK $\beta / N F-\kappa B$ disrupts adult hypothalamic neural stem cells to mediate a neurodegenerative mechanism of dietary obesity and pre-diabetes. Nat Cell Biol 14: 999-1012, 2012.

20. Williamson R, McNeilly A and Sutherland C: Insulin resistance in the brain: An old-age or new-age problem? Biochem Pharmacol 84: 737-745, 2012.

21. Hallschmid M and Schultes B: Central nervous insulin resistance: A promising target in the treatment of metabolic and cognitive disorders? Diabetologia 52: 2264-2269, 2009.

22. de la Monte SM: Insulin resistance and Alzheimer's disease. BMB Rep 42: 475-481, 2009.

23. S Roriz-Filho J, Sá-Roriz TM, Rosset I, Camozzato AL, Santos AC, Chaves ML, Moriguti JC and Roriz-Cruz M: (Pre) diabetes, brain aging, and cognition. Biochim Biophys Acta 1792: 432-443, 2009.

24. Driscoll I, Beydoun MA, An Y, Davatzikos C, Ferrucci L, Zonderman AB and Resnick SM: Midlife obesity and trajectories of brain volume changes in older adults. Hum Brain Mapp 33: 2204-2210, 2012

25. Kaidanovich-Beilin O, Cha DS and McIntyre RS: Crosstalk between metabolic and neuropsychiatric disorders. F1000 Biol Rep 4: 14, 2012.

26. Morris JK, Zhang H, Gupte AA, Bomhoff GL, Stanford JA and Geiger PC: Measures of striatal insulin resistance in a 6-hydroxydopamine model of Parkinson's disease. Brain Res 1240: 185-195, 2008.

27. Koren R, Toledano Y and Hod M: The use of insulin detemir during pregnancy: A safety evaluation. Expert Opin Drug Saf 14: 593-599, 2015.

28. Fantasia HC: Inhaled insulin - a new delivery for an old drug. Nurs Womens Health 19: 67-70, 2015.

29. Ford RJ, Fullerton MD, Pinkosky SL, Day EA, Scott JW, Oakhill JS, Bujak AL, Smith BK, Crane JD, Blümer RM, et al: Metformin and salicylate synergistically activate liver AMPK, inhibit lipogenesis and improve insulin sensitivity. Biochem J 468: 125-132, 2015.

30. White JR Jr: Empagliflozin, an SGLT2 inhibitor for the treatment of type 2 diabetes mellitus: A review of the evidence. Ann Pharmacother 49: 582-598, 2015.

31. Ramadan WH and Kabbara WK: Sitagliptin/Simvastatin: A first combination tablet to treat type 2 diabetes and hypercholesterolemia - a review of its characteristics. Vasc Health Risk Manag 11: 125-132, 2015.

32. Pacini G, Mari A, Fouqueray P, Bolze S and Roden M: Imeglimin increases glucose-dependent insulin secretion and improves $\beta$-cell function in patients with type 2 diabetes. Diabetes Obes Metab 17: 541-545, 2015.

33. Uchida S, Mitani A, Gunji E, Takahashi T and Yamamoto K: In vitro characterization of luseogliflozin, a potent and competitive sodium glucose co-transporter 2 inhibitor: Inhibition kinetics and binding studies. J Pharmacol Sci 128: 54-57, 2015.

34. Powell J, Miller SA and Taylor JR: Sodium-glucose cotransporter 2 inhibitors: The new option for diabetes mellitus management. South Med J 108: 82-90, 2015.

35. Hedrington MS and Davis SN: Ipragliflozin, a sodium-glucose cotransporter 2 inhibitor, in the treatment of type 2 diabetes. Expert Opin Drug Metab Toxicol 11: 613-623, 2015.

36. Yanai H, Adachi $H$, Katsuyama $H$, Moriyama S, Hamasaki $H$ and Sako A: Causative anti-diabetic drugs and the underlying clinical factors for hypoglycemia in patients with diabetes. World J Diabetes 6: 30-36, 2015.

37. Tran L, Zielinski A, Roach AH, Jende JA, Householder AM, Cole EE, Atway SA, Amornyard M, Accursi ML, Shieh SW, et al: Pharmacologic treatment of type 2 diabetes: oral medications. Ann Pharmacother 49: 540-556, 2015. 
38. Zheng J, Woo SL, Hu X, Botchlett R, Chen L, Huo Y and Wu C: Metformin and metabolic diseases: A focus on hepatic aspects Front Med 9: 173-186, 2015.

39. Agid Y: Parkinson's disease: Pathophysiology. Lancet 337: 1321-1324, 1991.

40. Špolcová A, Mikulášková B, Holubová M, Nagelová V, Pirnik Z, Zemenová J, Haluzík M, Železná B, Galas MC and Maletínská L: Anorexigenic lipopeptides ameliorate central insulin signaling and attenuate tau phosphorylation in hippocampi of mice with monosodium glutamate-induced obesity. J Alzheimers Dis 45: 823-835, 2015.

41. Priyadarshini M, Kamal MA, Greig NH, Realef $M$, Abuzenadah AM, Chaudhary AG and Damanhouri GA: Alzheimer's disease and type 2 diabetes: Exploring the association to obesity and tyrosine hydroxylase. CNS Neurol Disord Drug Targets 11: 482-489, 2012.
42. Picone P, Nuzzo D, Caruana L, Messina E, Barera A, Vasto S and Di Carlo M: Metformin increases APP expression and processing via oxidative stress, mitochondrial dysfunction and $\mathrm{NF}-\kappa \mathrm{B}$ activation: Use of insulin to attenuate metformin's effect. Biochim Biophys Acta 1853: 1046-1059, 2015.

43. Miller DB and O'Callaghan JP: Do early-life insults contribute to the late-life development of Parkinson and Alzheimer diseases? Metabolism 57 (Suppl 2): S44-S49, 2008

44. Song I, Muller C, Louw J and Bouwens L: Regulating the beta cell mass as a strategy for type-2 diabetes treatment. Curr Drug Targets 16: 516-524, 2015 . 